\title{
Comparative Effectiveness of Digital Breast Tomosynthesis and Mammography in Older Women
}

\author{
Akhil Upneja' ${ }^{7}$, Jessica B. Long ${ }^{2,3}$, Jenerius A. Aminawung ${ }^{2,3}$, Kelly A. Kyanko ${ }^{4}$, \\ Natalia Kunst ${ }^{3,5}$, Xiao Xu' $^{6}$, Susan H. Busch7 , Cary P. Gross ${ }^{2,3}$, and llana B. Richman ${ }^{2,3}$ (B)
}

${ }^{1}$ Yale School of Medicine, New Haven, CT, USA; ${ }^{2}$ Department of Internal Medicine, Section of General Internal Medicine, Yale School of Medicine, New Haven, CT, USA; ${ }^{3}$ Cancer Outcomes, Public Policy, and Effectiveness Research (COPPER) Center, Yale Cancer Center and Yale University School of Medicine, New Haven, CT, USA; ${ }^{4}$ Department of Population Health, New York University School of Medicine, New York, NY, USA; ${ }^{5} \mathrm{Harvard}$ Medical School and Harvard Pilgrim Health Care Institute, Boston, MA, USA; ${ }^{6}$ Department of Obstetrics and Gynecology, Yale School of Medicine, New Haven, CT, USA; ${ }^{7}$ Department of Health and Policy Management, Yale School of Public Health, New Haven, CT, USA.

BACKGROUND: Digital breast tomosynthesis (DBT) has become a prevalent mode of breast cancer screening in recent years. Although older women are commonly screened for breast cancer, little is known about screening outcomes using DBT among older women.

OBJECTIVE: To assess proximal screening outcomes with DBT compared to traditional two-dimensional(2-D) mammography among women 67-74 and women 75 and older.

DESIGN: Cohort study.

PARTICIPANTS: Medicare fee-for-service beneficiaries aged 67 years and older with no history of prior cancer who received a screening mammogram in 2015.

MAIN MEASURES: Use of subsequent imaging (ultrasound and diagnostic mammography) as an indication of recall, breast cancer detection, and characteristics of breast cancer at the time of diagnosis. Analyses used weighted logistic regression to adjust for potential confounders.

KEY RESULTS: Our study included 26,406 women aged 67-74 and 17,001 women 75 and older who were screened for breast cancer. Among women 75 and older, the rate of subsequent imaging among women screened with DBT did not differ significantly from 2-D mammography (91.8 versus 97.0 per 1,000 screening mammograms, $p=0.37$ ). In this age group, DBT was associated with 2.1 additional cancers detected per 1,000 screening mammograms compared to $2 \mathrm{D}$ (11.5 versus 9.4 , $p=0.003$ ), though these additional cancers were almost exclusively in situ and stage I invasive cancers. For women 67-74 years old, DBT was associated with a higher rate of subsequent imaging than 2-D mammography (113.9 versus $100.3, p=0.004)$ and a higher rate of stage I

Prior Presentations An earlier version of this work was presented in poster format at the Society of General Internal Medicine New England Regional Meeting on November 2, 2019. A poster presentation of this work was uploaded as a part of the conference materials for the Society of General Internal Medicine Annual Meeting in May 2020 in lieu of an oral presentation due to the COVID-19 pandemic. A virtual oral presentation of this work was presented at the Yale Cancer Center Trainee Colloquium on June 5, 2020.

Received March 8, 2021

Accepted September 2, 2021

Published online September 30, 2021 invasive cancer detection ( 4.7 versus $3.7, p=0.002$ ), but not other stages.

CONCLUSIONS: Breast cancer screening with DBT was not associated with lower rates of subsequent imaging among older women. Most additional cancers detected with DBT were early stage. Whether detecting these additional early-stage cancers among older women improves health outcomes remains uncertain.

KEY WORDS: comparative effectiveness; digital breast tomosynthesis; mammography; breast cancer; older women.

J Gen Intern Med 37(8):1870-6

DOI: $10.1007 / \mathrm{s} 11606-021-07132-6$

(c) Society of General Internal Medicine 2021

\section{INTRODUCTION}

Prior to 2011, two-dimensional(2-D) digital mammography was the standard of care for breast cancer screening. In 2011, digital breast tomosynthesis (DBT), an imaging modality that generates 3-dimensional tomographic images of the breast, was FDA-approved and has since been widely adopted. In one recent study, DBT was used in over $40 \%$ of screening examinations, and as of January 2021, nearly $75 \%$ of certified breast imaging facilities in the USA have DBT units. ${ }^{1,2}$ Several observational studies have demonstrated that women screened with DBT are less likely to be called back for additional diagnostic imaging compared to women screened with 2-D mammography, a measure known as recall. ${ }^{3-7}$ Other studies have shown higher cancer detection rates when DBT is used over 2-D mammography. ${ }^{8-13}$

Some studies have examined screening outcomes using DBT compared to 2-D mammography specifically among older women. One recent study found that among women over the age of 65, DBT had a lower abnormal interpretation rate, a higher specificity, and a similar cancer detection rate compared with 2-D mammography. ${ }^{14}$ A second study reported lower recall rates with DBT among women 70 and older. ${ }^{15}$ However, neither study reported outcomes among women 75 and older. This age group is of particular interest because mammography itself is of uncertain benefit for women over 
the age of 74. No randomized trials of mammography have included women over 74, and a recent study found no difference in 8 -year breast cancer mortality between women over 75 who received annual 2-D screening mammography and those who did not. ${ }^{16}$ The US Preventive Services Task Force guidelines give screening among women 75 and older an "I" rating, indicating that there is insufficient evidence for or against screening. ${ }^{17,18}$ Despite the dearth of evidence for effectiveness in older women, more than $50 \%$ of women over the age of 75 continue to receive screening mammography. ${ }^{16}$ Whether or not to deploy DBT - a newer, more expensive technology — in this age group is unclear.

Given ongoing uncertainty about the effectiveness of DBT for screening among older women, the goal of this study was to evaluate proximal screening outcomes, including subsequent imaging use and biopsy rates, cancer detection rates, and characteristics of detected cancers, for older women who were screened with 2-D mammography and DBT. Our study focused on screening outcomes among women 75 and older but also evaluated outcomes among women $67-74$ as an important comparison group.

\section{METHODS}

\section{Data Source}

We used data from the Surveillance, Epidemiology, and End Results Program-Medicare linked database (SEER-Medicare). SEER-Medicare integrates Medicare claims with data on cancer diagnosis for patients enrolled in the SEER registry. We used two samples to construct the study cohort: (1) the SEER cancer sample, which includes all Medicare beneficiaries reported to SEER with a diagnosis of breast cancer through calendar year 2015, and (2) the SEER "non-cancer" sample, a 5\% representative sample of Medicare beneficiaries living in SEER regions who have not been diagnosed with cancer through the end of available follow-up. We combined these samples, weighting the non-cancer sample 20:1 to balance the differential sampling between the cancer sample, which includes all cases, and the non-cancer sample, which is a 5\% sample. This approach constructs a representative population of Medicare beneficiaries residing in SEER areas in 2015. ${ }^{19}$ The Yale Human Investigation Committee determined that this study did not constitute human subjects research.

Study sample. Among women continuously enrolled in Medicare Fee for Service parts A \& B from January 1, 2013, to December 31, 2016, we identified women aged 67 and older who received a screening mammogram between January 1 and December 31, 2015, the first year that DBT was covered in the Medicare program. We excluded women who had a history of breast cancer or other cancers prior to screening mammography, based on either SEER data or Medicare claims. We also excluded women with genetic cancer syndromes and women who underwent prophylactic mastectomy prior to mammography (eTable 1). Finally, we excluded women whose cancer was identified by death certificate or autopsy or had missing diagnosis dates within SEER. For analyses focusing on cancer diagnosis, we included women screened between January 1, 2015, and August 31, 2015, rather than the full calendar year. This was to allow for 4 months of follow-up in 2015 during which we could observe cancer diagnoses after screening.

Variables. The primary predictor of interest was use of 2-D mammography or DBT for breast cancer screening. 2-D mammography was defined as screening with film or full-field digital mammography, with or without computer-aided detection. We identified 2-D screening mammograms using a validated algorithm which we adapted for use with codes from the $10^{\text {th }}$ revision of the International Statistical Classification of Diseases and Related Health Problems (ICD-10) (eMethods). ${ }^{20}$ DBT is typically performed and billed in conjunction with 2-D mammography for women enrolled in Medicare. Therefore, we defined screening DBT as receipt of a 2-D screening mammogram, as defined by the algorithm, in conjunction with a claim for screening DBT (eTable 1).

Women screened with DBT may have important clinical and demographic differences from women screened with 2-D. Thus, we compared patient characteristics including age, race, eligibility for state-sponsored insurance (e.g., Medicaid programs), previous breast cancer screening history, family history of breast cancer, and comorbidities by screening type (DBT or 2-D). ${ }^{21}$ Of note, women of Hispanic ethnicity are included in all categories of race. Covariates were identified from SEER data and from claims. We used data on neighborhood poverty from the American Community Survey 20082012 aggregated at the zip code level as a proxy for individual income. $^{22}$

We evaluated downstream testing including imaging and biopsy after screening DBT or 2-D mammography among women screened between January 1, 2015, and December 31, 2015. We defined "subsequent imaging" as the presence of a claim for diagnostic mammography, with or without DBT, or unilateral or focused breast ultrasound, conducted in the 4 months after the index mammogram. This definition is intended to capture women who return for follow-up imaging after an abnormal mammogram. We considered bilateral whole breast ultrasound to be likely screening and reported it separately as a covariate. We also evaluated use of MRI and biopsy after DBT and 2-D mammography, but did not include MRI within our definition of subsequent imaging, as MRI is often used either to screen high-risk women or to evaluate a newly diagnosed breast cancer.

We evaluated cancer diagnoses among the subset of women screened between January 1, 2015, and August 31, 2015. This period was chosen to allow 4 months of follow-up to assess for the presence of a cancer diagnosis after breast cancer screening, since the most current data at the time of study included data on cancer diagnoses only through December 31, 2015. We defined screen-detected cancer diagnosis as any breast 
cancer diagnosis, including carcinoma in situ, within 4 months of a screening mammogram. We evaluated stage at diagnosis as defined by the $6^{\text {th }}$ edition of the AJCC staging system and tumor size. ${ }^{23}$ Using a previously established classification system, we also grouped tumors into three prognostic categories: biologically favorable, intermediate, or unfavorable based on tumor grade and hormone receptor status (eTable 2) ${ }^{24}$ For consistency, we also assessed imaging and biopsy outcomes in the subset of women screened between January 1, 2015, and August 31, 2015, i.e., the subset of women for whom we were also able to evaluate cancer diagnoses.

Lastly, we calculated positive predictive values following subsequent imaging and biopsy, defined as the proportion of women diagnosed with cancer from among those who underwent subsequent imaging or biopsy after an initial screening exam. Procedure codes used to define imaging and testing outcomes are detailed in eTable 1.

\section{Statistical Analysis}

We stratified all analyses by two age groups, 67-74 and over 75 , to assess for differences in outcomes between the younger age group, for which data support the use of screening mammography, and the older age group, where benefit is less clear. We also stratified analyses by quarter to evaluate screening outcomes over time. We used weighted chi-square tests to evaluate the differences in clinical and demographic characteristics between women screened with DBT and 2-D mammography. We used weighted logistic regression to evaluate the relationship between screening type and subsequent testing. For cancer stage, tumor size, and biologic category, we used weighted multinomial logistic regression to account for multiple outcome categories. Analyses were adjusted for age, race, Elixhauser comorbidity score, family history of breast cancer, number of screening mammograms in 2013-2014, dual eligibility, SEER area, and zip code-level prevalence of poverty. We also adjusted for influenza vaccination and PCP visits as markers of a patient's use of preventive services and access to care overall, which could confound their use of DBT and use of downstream imaging. Lastly, we adjusted for use of screening ultrasound (defined as bilateral, whole breast ultrasound), as it may be a marker of risk and may also affect screening outcomes. Models used robust standard errors, clustered by SEER area. We used predicted probabilities calculated at observed values to express adjusted outcomes per 1,000 women screened.

\section{RESULTS}

Our final sample included 26,406 women ages 67-74 and 17,001 women ages 75 and older who were screened for breast cancer during 2015, representing a weighted population size of 754,568 (eTable 3). Overall, 79\% of women received 2-D mammography and $21 \%$ received DBT, with use of DBT increasing over time (eFigure 1). Among women 75 and older,
DBT use was more common in wealthier zip codes $(26 \%$ in the wealthiest zip codes versus $14 \%$ in the poorest zip codes, $p<0.001)$ and less common among Black versus White women (14\% versus $21 \%$, respectively, $p<0.001)$. Women with eligibility for state-sponsored health coverage were also less likely to receive DBT than those with Medicare only (12\% versus $21 \%, p<0.001)$. Patterns of DBT use were generally similar among women aged 67-74(Table 1).

For women 75 and older, adjusted rates of subsequent imaging were not significantly different between those screened with DBT and 2-D mammography (91.8 versus 97.0 per 1,000 mammograms with DBT versus $2-\mathrm{D}, p=0.37$; Table 2). By contrast, for women aged 67-74, adjusted rates of subsequent imaging were higher following DBT than following 2-D mammography (113.9 versus 100.3 women with subsequent imaging per 1,000 mammograms, $p=0.004$; Table 2). In both age groups, point estimates for subsequent imaging among women screened with DBT were highest in the first quarter of the year and declined over time (eTable $4 \mathrm{a}$, eFigure 2a). Congruent with this finding, in the subset of women screened between January and August 2015, unadjusted subsequent imaging rates were higher with DBT, both for women 67-74 (120.4 versus 99.6 per $1000, p=0.001)$ and for women 75 and older (109.7 versus 93.3, $p=0.03$ ) (eTable 5).

Among women 75 and older, adjusted biopsy rates were not significantly different between women screened with DBT and those screened with 2-D mammography (18.9 versus 16.8 per 1,000 mammograms, $p=0.30$; Table 2). Among women $67-74$, biopsy rates were higher among women screened with DBT (21.9 versus 16.1 per 1,000 mammograms, $p<0.001$; Table 2).

We evaluated rates of cancer detection in the subset of women screened between January $1^{\text {st }}$ and August $31^{\text {st }}$, 2015 $(n=28,626)$, representing a weighted population size of 490,307. Among women 75 and older, DBT was associated with a higher overall rate of cancer detection compared to 2-D mammography ( 11.5 versus 9.4 cancers per 1,000 mammograms, $p=0.003$; Table 3 ) after adjusting for covariates. Women screened with DBT were more likely to be diagnosed with an in situ neoplasm (2.5 versus 1.5 per 1,000 mammograms, $p<0.001$; Table 3 ) or an invasive stage I cancer (6.0 versus 4.9 per 1,000 mammograms, $p=0.013$; Table 3 ), but not a more advanced cancer. In contrast, for women 67 74 , the adjusted overall cancer detection rate was not significantly different between women screened with DBT versus 2D mammography (8.1 versus 7.3 per 1,000 mammograms, $p=0.056$; Table 3). However, DBT was associated with a higher rate of invasive stage I cancer detection (4.7 versus 3.7 per 1,000 mammograms, $p=0.002)$. Unadjusted cancer detection rates were similar to adjusted rates (eTable 6). Point estimates for cancer detection were generally stable over time (eTable $4 b$, eFigure $2 b$ ).

Among women 75 and older, there were no significant differences in biological features of cancers detected among 
Table 1 Clinical and Demographic Characteristics of Women Screened with 2-D Mammography Versus DBT

\begin{tabular}{|c|c|c|c|c|c|c|c|}
\hline & \multicolumn{3}{|l|}{$67-74$} & \multicolumn{3}{|l|}{$75+$} & \multirow[t]{3}{*}{$p$ value for interaction ${ }^{\S}$} \\
\hline & \multirow{2}{*}{$\frac{2-\mathrm{D}}{N(\%)}$} & \multirow{2}{*}{$\frac{\text { DBT }}{N(\%)}$} & \multirow[t]{2}{*}{$p^{*}$} & \multirow{2}{*}{$\frac{2-\mathrm{D}}{N(\%)}$} & \multirow{2}{*}{$\frac{\text { DBT }}{N(\%)}$} & \multirow[t]{2}{*}{$p^{\ddagger}$} & \\
\hline & & & & & & & \\
\hline Total sample (weighted) & $364,254(78)$ & $100,539(22)$ & & $230,685(80)$ & $59,090(20)$ & & \\
\hline \multicolumn{8}{|l|}{ Age group } \\
\hline $67-69$ & $156,500(78)$ & $44,331(22)$ & \multirow[t]{5}{*}{$<0.001$} & - & - & \multirow[t]{5}{*}{$<0.001$} & \multirow[t]{5}{*}{-} \\
\hline $70-74$ & $207,754(79)$ & $56,208(21)$ & & - & - & & \\
\hline $75-79$ & - & - & & $132,064(80)$ & $33,913(20)$ & & \\
\hline $80-84$ & - & - & & $67,119(79)$ & $17,444(21)$ & & \\
\hline $85+$ & - & - & & $31,502(80)$ & $7,733(20)$ & & \\
\hline \multicolumn{8}{|l|}{ Race } \\
\hline White & $302,478(77)$ & $88,467(23)$ & \multirow[t]{3}{*}{$<0.001$} & $196,256(79)$ & $53,091(21)$ & \multirow[t]{3}{*}{$<0.001$} & \multirow[t]{3}{*}{0.13} \\
\hline Black & $29,171(84)$ & $5,543(16)$ & & $16,961(86)$ & 2,849 (14) & & \\
\hline Other & $32,605(83)$ & $6,529(17)$ & & $17,468(85)$ & $3,150(15)$ & & \\
\hline \multicolumn{8}{|c|}{ Percent in zip code at or below poverty } \\
\hline$<5 \%$ & $51,615(73)$ & $18,714(27)$ & \multirow[t]{5}{*}{$<0.001$} & $32,762(74)$ & $11,524(26)$ & \multirow[t]{5}{*}{$<0.001$} & \multirow[t]{5}{*}{$<0.001$} \\
\hline $5-9.9 \%$ & $111,751(76)$ & $36,032(24)$ & & $71,683(78)$ & $20,804(22)$ & & \\
\hline $10-19.9 \%$ & $126,781(80)$ & $31,589(20)$ & & $79,323(81)$ & $18,764(19)$ & & \\
\hline$\geq 20 \%$ & $67,483(84)$ & $12,674(16)$ & & $43,914(86)$ & $7,350(14)$ & & \\
\hline Ünknown & $6,624(81)$ & $1,530(19)$ & & $3,003(82)$ & $648(18)$ & & \\
\hline \multicolumn{8}{|c|}{ Family history of breast cancer } \\
\hline Yes & $30,984(70)$ & $13,068(30)$ & \multirow{2}{*}{$<0.001$} & $20,965(74)$ & $7,538(26)$ & \multirow{2}{*}{$<0.001$} & \multirow{2}{*}{$<0.001$} \\
\hline No & $333,270(79)$ & $87,471(21)$ & & $209,720(80)$ & $51,552(20)$ & & \\
\hline \multicolumn{8}{|c|}{ State-sponsored health coverage } \\
\hline Eligible & $37,902(88)$ & $5,176(12)$ & \multirow[t]{2}{*}{$<0.001$} & $23,389(88)$ & $3,205(12)$ & $<0.001$ & 0.001 \\
\hline Non-eligible & $326,352(77)$ & $95,363(23)$ & & 207,296 (79) & $55,885(21)$ & & \\
\hline Screens in past 2 years & & & & & & & \\
\hline 0 & $81,368(81)$ & $18,764(19)$ & $<0.001$ & $44,661(81)$ & $10,141(19)$ & $<0.001$ & $<0.001$ \\
\hline 1 & $275,015(78)$ & $78,875(22)$ & & $180,506(79)$ & $46,756(21)$ & & \\
\hline 2 & $7,871(73)$ & $2,900(27)$ & & $5,518(72)$ & $2,193(28)$ & & \\
\hline Comorbidity & & & & & & & \\
\hline 0 & $153,070(77)$ & $46,468(23)$ & $<0.001$ & $71,351(78)$ & $20,181(22)$ & $<0.001$ & 0.001 \\
\hline 1 to 2 & $151,884(79)$ & $40,648(21)$ & & $101,160(79)$ & $26,467(21)$ & & \\
\hline $3+$ & $59,300(82)$ & $13,423(18)$ & & $58,174(82)$ & $12,442(18)$ & & \\
\hline Screening ultrasound & & & & & & & \\
\hline Yes & $3,601(58)$ & $2,601(42)$ & $<0.001$ & $1,975(63)$ & 1,179 & $<0.001$ & 0.001 \\
\hline No & $360,653(79)$ & $97,938(21)$ & & $228,710(80)$ & $57,911(20)$ & & \\
\hline
\end{tabular}

${ }^{7} p$ value evaluates group differences between women screened with DBT and 2-D mammography

${ }^{s} p$ value for interaction evaluates interaction between characteristics and age group

$D B T$, digital breast tomosynthesis; 2-D, 2-D mammography

women screened with DBT versus 2-D mammography (Table 3). Among women 67-74, DBT was associated with a higher cancer detection rate for cancers with favorable biologic features (2.1 versus 1.7 per 1,000 mammograms, $p=0.036$ ), but not intermediate or unfavorable biologic features (Table 3 ).

Table 2 Follow-up Imaging and Testing Rates for Women Screened with 2-D Mammography Versus DBT, per 1,000 Women Screened

\begin{tabular}{|c|c|c|c|c|c|c|c|}
\hline \multirow[t]{2}{*}{$\overline{\text { Outcome }}$} & \multicolumn{3}{|l|}{$67-74$} & \multicolumn{3}{|l|}{$75+$} & \multirow[t]{2}{*}{$p^{*}$} \\
\hline & $2-\mathrm{D}(95 \% \mathrm{CI})$ & DBT (95\% CI) & $p$ & 2-D (95\% CI) & DBT (95\% CI) & $p$ & \\
\hline Total sample* (weighted) & 364,254 & 100,539 & & 230,685 & 59,090 & & \\
\hline \multicolumn{8}{|l|}{ Unadjusted } \\
\hline Subsequent imaging & $99.5(95.2-104)$ & $117.0(108.3-125.6)$ & $<0.001$ & $95.8(90.6-101)$ & $96.3(86.1-106.4)$ & 0.93 & 0.034 \\
\hline Diagnostic mammography & $69.2(65.6-72.7)$ & $65.1(58.6-71.6)$ & 0.283 & $68.0(63.6-72.4)$ & $58.8(50.9-66.7)$ & 0.056 & 0.38 \\
\hline Diagnostic ultrasound & $69.7(66.1-73.3)$ & $92.1(84.3-99.8)$ & $<0.001$ & $64.4(60.2-68.6)$ & $73.4(64.4-82.3)$ & 0.065 & 0.083 \\
\hline MRI & $3.79(3.22-4.36)$ & $5.67(4.22-7.12)$ & 0.008 & $2.09(1.65-2.54)$ & $2.45(1.69-3.22)$ & 0.41 & 0.32 \\
\hline Biopsy & $16.1(14.7-17.6)$ & $21.8(18.5-25.1)$ & 0.001 & $16.8(15.1-18.5)$ & $18.6(15.1-22.2)$ & 0.35 & 0.17 \\
\hline \multicolumn{8}{|l|}{ Adjusted $^{\S}$} \\
\hline Subsequent imaging & $100.3(96.1-104.5)$ & $113.9(105.4-122.4)$ & 0.004 & $97.0(91.8-102)$ & $91.8(82.0-102)$ & 0.37 & 0.038 \\
\hline Diagnostic mammography & $69.1(65.6-72.7)$ & $65.3(58.7-71.8)$ & 0.32 & $68.5(64.1-72.9)$ & $57.2(49.3-65.0)$ & 0.021 & 0.41 \\
\hline Diagnostic ultrasound & $70.5(67.0-74.1)$ & $88.9(81.3-96.5)$ & $<0.001$ & $65.3(61.0-69.5)$ & $69.8(61.2-78.3)$ & 0.35 & 0.093 \\
\hline MRI & $3.89(3.29-4.48)$ & $5.22(3.82-6.62)$ & 0.066 & $2.11(1.67-2.55)$ & $2.38(1.64-3.13)$ & 0.51 & 0.34 \\
\hline Biopsy & $16.1(14.7-17.6)$ & $21.9(18.5-25.3)$ & $<0.001$ & $16.8(15.0-18.5)$ & $18.9(15.2-22.5)$ & 0.30 & 0.18 \\
\hline
\end{tabular}

*Includes women screened January-December 2015

"Indicates $p$ value for interaction between age groups

${ }^{s}$ Adjusted for age, race, local area poverty, family history of breast cancer, state-sponsored health coverage eligibility, prior screening mammogram, comorbidity burden, screening ultrasound, receipt of a flu shot, recent primary care visit, SEER region or state (state for CA and GA)

$D B T$, digital breast tomosynthesis; 2-D, 2-D mammography 
Table 3 Cancer Detection Rates from SEER for Women Screened with 2-D Versus DBT, per 1,000 Women Screened

\begin{tabular}{|c|c|c|c|c|c|c|c|}
\hline \multirow[t]{2}{*}{$\overline{\text { Outcomes }^{\dagger}}$} & \multicolumn{3}{|l|}{$67-74$} & \multicolumn{3}{|l|}{$75+$} & \multirow[t]{2}{*}{$\overline{p^{*}}$} \\
\hline & 2-D (95\% CI) & DBT (95\% CI) & $p$ & $2-D(95 \%$ CI $)$ & DBT $(95 \%$ CI) & $p$ & \\
\hline Total sample* (weighted) & 240,851 & 58,816 & & 156,294 & 34,346 & & \\
\hline Total cancer diagnoses & $7.3(6.9-7.6)$ & $8.1(7.3-8.9)$ & 0.056 & $9.4(8.8-9.9)$ & $11.5(10.1-12.8)$ & 0.003 & 0.45 \\
\hline In situ cancers & $1.5(1.3-1.7)$ & $1.6(1.3-1.9)$ & 0.61 & $1.5(1.3-1.7)$ & $2.5(2.0-3.1)$ & $<0.001$ & 0.059 \\
\hline Total invasive cancer diagnoses & $6.2(5.9-6.6)$ & $6.9(6.2-7.7)$ & 0.11 & $8.4(7.9-8.9)$ & $9.4(8.2-10.6)$ & 0.13 & 0.98 \\
\hline Stage 1 & $3.7(3.5-4.0)$ & $4.7(4.1-5.3)$ & 0.002 & $4.9(4.5-5.3)$ & $6.0(5.1-6.9)$ & 0.013 & 0.97 \\
\hline Stage 2 & $1.6(1.5-1.8)$ & $1.5(1.1-1.8)$ & 0.44 & $2.3(2.0-2.5)$ & $2.1(1.5-2.6)$ & 0.50 & 0.77 \\
\hline Stage 3 & $0.29(0.22-0.36)$ & $0.31(0.15-0.47)$ & 0.84 & $0.32(0.23-0.41)$ & $0.43(0.18-0.68)$ & 0.36 & 0.58 \\
\hline Stage 4 & $0.09(0.05-0.10)$ & $0.03(0.01-0.08)$ & 0.13 & $0.18(0.12-0.25)$ & $0.16(0.02-0.30)$ & 0.79 & 0.41 \\
\hline Invasive tumor size $<2 \mathrm{~cm}$ & $4.1(3.8-4.3)$ & $5.0(4.4-5.6)$ & 0.005 & $5.2(4.8-5.6)$ & $6.5(5.6-7.4)$ & 0.056 & 0.66 \\
\hline Invasive tumor size $2-5 \mathrm{~cm}$ & $1.5(1.3-1.6)$ & $1.3(1.0-1.6)$ & 0.36 & $2.2(2.0-2.5)$ & $2.0(1.5-2.5)$ & 0.43 & 0.79 \\
\hline Invasive tumor size $>5 \mathrm{~cm}$ & $0.20(0.14-0.26)$ & $0.21(0.08-0.34)$ & 0.91 & $0.29(0.20-0.37)$ & $0.26(0.06-0.46)$ & 0.82 & 0.78 \\
\hline Biologically ${ }^{\S}$ favorable & $1.7(1.5-1.8)$ & $2.1(1.7-2.5)$ & 0.036 & $2.3(2.1-2.6)$ & $2.6(2.0-3.2)$ & 0.34 & 0.72 \\
\hline Biologically intermediate & $3.1(2.9-3.3)$ & $3.5(3.0-4.0)$ & 0.20 & $4.2(3.9-4.6)$ & $4.9(4.1-5.7)$ & 0.12 & 0.99 \\
\hline Biologically unfavorable & $0.73(0.62-0.84)$ & $0.76(0.52-0.99)$ & 0.83 & $0.88(0.73-1.03)$ & $1.02(0.66-1.40)$ & 0.44 & 0.72 \\
\hline PPV subsequent imaging (\%) & $7.0(6.5-7.5)$ & $7.2(6.2-8.2)$ & 0.69 & $9.7(8.8-10.5)$ & $10.8(8.9-12.7)$ & 0.26 & 0.85 \\
\hline PPV biopsy $(\%)$ & $42.2(37.7-46.8)$ & $41.5(33.6-49.4)$ & 0.87 & $53.6(47.8-59.5)$ & $50.6(38.6-62.7)$ & 0.66 & 0.92 \\
\hline
\end{tabular}

${ }^{\dagger}$ Adjusted for age, race, local area poverty, family history of breast cancer, state-sponsored health coverage eligibility, prior screening mammogram, comorbidity burden, screening ultrasound, receipt of a flu shot, recent primary care visit, SEER region or state (state for CA and GA)

*Includes women screened January-August 2015

Indicates $p$ value for interaction between age groups

${ }^{\S}$ Biological prognostic categories are based on hormone receptor status and grade

SEER, Surveillance, Epidemiology, and End Results Program-Medicare linked database; PPV, positive predictive value

Among women 75 and older, positive predictive values after subsequent imaging or biopsy for DBT were not statistically different from those for 2-D mammography $(10.8 \%$ versus $9.7 \%$ for subsequent imaging, $p=0.26 ; 50.6 \%$ versus $53.6 \%$ for biopsy, $p=0.66$; Table 3 ). We observed similar results for positive predictive values of subsequent imaging and biopsy among women 67-74 years old (Table 3 ).

\section{DISCUSSION}

In this study comparing short-term breast cancer screening outcomes among older women screened with DBT or 2-D mammography, we found that DBT was not associated with lower subsequent imaging rates compared to 2-D mammography but was associated with higher rates of early-stage breast cancer diagnosis.

Our findings differ from several studies which have shown that DBT is associated with lower recall rates compared to 2-D screening mammography. ${ }^{5,6,8}$ Lower recall has been held up as a major advantage of DBT, as further workup creates additional anxiety and expense. We have shown that DBT is not associated with use of less subsequent imaging among older women, suggesting that this potential advantage has not been realized in this group.

There are several reasons that may account for the difference between our findings and some prior studies. First, we evaluated DBT at a time when the technology was still relatively new. Indeed, consistent with other studies, we found that the point estimates for subsequent imaging rates among women screened with DBT declined over the course of a calendar year, suggesting some improvement in performance over time. ${ }^{3}$ Longer periods of follow-up will be needed to fully characterize DBT performance among older women.

A second possible explanation is that DBT affords less of an advantage over 2-D mammography in older women. The performance of 2-D mammography itself improves with age and some studies have suggested a larger absolute reduction in recall with DBT among younger women compared to older women. ${ }^{25,5,15}$ However, not all studies have shown consistent age effects, and there are important remaining questions about how age, breast density, and screening round may all impact DBT performance among older women. ${ }^{26}$

Consistent with prior studies, we observed that DBT was associated with higher rates of cancer detection compared to 2D mammography. ${ }^{8-12}$ Additional cancers detected were uniformly small, early-stage cancers. Among women 75 and older, DBT was also associated with higher rates of in situ diagnosis, and among women 67-74, DBT was associated with a higher rate of detection of biologically favorable cancers.

Although no long-term studies have evaluated whether the higher cancer detection rate observed with DBT improves mortality, several studies have projected long-term benefits of DBT using simulation modeling. These studies suggest that DBT may avert approximately one additional breast cancer death for every $2000-5000$ women screened for at least 10 years, compared to 2-D mammography. However, these studies focus on younger populations and women with dense breasts. ${ }^{27,28}$ Among older women, the higher cancer detection rate of DBT is of uncertain benefit. Diagnosis of early-stage or in situ breast cancer has not been shown to improve life expectancy among older women and it is not clear whether DBT's higher cancer detection rate will ultimately improve outcomes for older women. ${ }^{29,30}$ In addition, increased cancer 
detection may contribute to harm from overdiagnosis and overtreatment. Thus, as we use more sensitive technologies for breast cancer screening, we will need better data to understand whether older women benefit and alternate approaches to managing lower risk cancers to avoid harm from overtreatment in older women.

We acknowledge several limitations of this study. First, though we adjusted for potential confounders in our analysis, there may be other unmeasured differences between women who received DBT and 2-D mammography that contribute to our findings. In particular, higher-risk women or women with dense breasts may be preferentially referred for DBT, which may result in greater use of subsequent imaging and higher cancer detection rates. While we were unable to observe mammographic findings including breast density, we adjusted for age, which is strongly associated with breast cancer risk and breast density, as well as use of supplemental ultrasound, which may be more commonly used among women with dense breasts. Importantly, the incremental increase in cancer detection that we observed with DBT was similar in magnitude to what has been observed in studies able to adjust for breast density, supporting our findings. 5

Second, we used claims-based definitions of some variables, which have limitations. We modified a validated algorithm designed to distinguish screening mammograms from diagnostic mammograms. While our changes replaced outdated ICD-9 codes with their equivalent ICD-10 and CPT/ HCPCS codes, these modifications were not themselves validated. We also acknowledge possible limitations in evaluating subsequent imaging. Because we used claims data, we could not observe mammographic results including BIRADS classification and thus could not evaluate recall directly. However, we believe that even if use of subsequent imaging is not a perfect marker of recall, it is an important outcome in itself, as returning for follow-up imaging results in additional time, expense, and anxiety for screened women.

\section{CONCLUSIONS}

In summary, in this study of older women undergoing breast cancer screening, DBT was associated with similar or higher rates of subsequent imaging compared to 2-D mammography. While DBT was associated with higher cancer detection rates, the additional cancers detected were mostly early-stage or in situ tumors. Understanding the long-term outcomes and value of DBT in older women will help guide future recommendations for screening in this population.

Supplementary Information The online version contains supplementary material available at https://doi.org/10.1007/s11606-02107132-6.

Acknowledgements: All contributors to this manuscript met the criteria for authorship.
Corresponding Author: Ilana B. Richman, Department of Internal Medicine, Section of General Internal Medicine, Yale School of Medicine, New Haven, CT 06510, USA (e-mail: ilana.richman@yale. edu).

Funding This work was supported by NIH/NCATS KL2 TROO1862 (Richman), NIH/NCI KO8 CA238725 (Richman), NIH T35 AG04968504 (Upneja), and American Cancer Society RSGI-15-151-01 (Gross, Busch, and Xu). Drs. Richman and Kyanko report salary support from the Centers for Medicare and Medicaid Services to develop health care quality measures outside of the submitted work. Dr. Gross reports research grants from NCCN/Pfizer AstraZeneca, Johnson \& Johnson, and Genentech, and travel funding from Flatiron Health. Ms. Kunst reports funding from the Research Council of Norway and LINK Medical Research (grant numbers 276146 and 304034) during the conduct of the study, and personal fees for speaking from Thermo Fisher Scientific. Professor Busch reports research funding from NIDA, NIMH, and the Robert Wood Johnson Foundation. NIH was not involved in the study's design, conduct, or reporting. The content is solely the responsibility of the authors and does not necessarily represent the official views of the National Institutes of Health. The collection of the California cancer incidence data used in this study was supported by the California Department of Public Health as part of the statewide cancer reporting program mandated by California Health and Safety Code Section 103885; the National Cancer Institute's Surveillance, Epidemiology and End Results Program under contract NO1-PC-35136 awarded to the Northern California Cancer Center, contract NO1-PC-35139 awarded to the University of Southern California, and contract NO2-PC-15105 awarded to the Public Health Institute; and the Centers for Disease Control and Prevention's National Program of Cancer Registries, under agreement \#U55/CCR921930-02 awarded to the Public Health Institute. The ideas and opinions expressed herein are those of the author(s) and endorsement by the State of California Department of Public Health, the National Cancer Institute, and the Centers for Disease Control and Prevention or their Contractors and Subcontractors is not intended nor should be inferred. The authors acknowledge the efforts of the Applied Research Program, NCI; the Office of Research, Development and Information, CMS; Information Management Services (IMS), Inc.; and the Surveillance, Epidemiology, and End Results (SEER) Program tumor registries in the creation of the SEER-Medicare database. The interpretation and reporting of the SEER-Medicare data are the sole responsibility of the authors.

Data Availability The SEER-Medicare datasets used for this study are available to researchers at https://healthcaredelivery.cancer.gov/ seermedicare/obtain/.

\section{REFERENCES}

1. Richman IB, Hoag JR, Xu X, et al. (2019) Adoption of Digital Breast Tomosynthesis in Clinical Practice. JAMA Intern Med.

2. Mammography Quality Standards Act and Program: MQSA National Statistics. (2021) U.S. Food \& Drug Administration. Updated January 4, 2021. Accessed January 31, 2021.

3. Sprague BL, Coley RY, Kerlikowske K, et al. Assessment of Radiologist Performance in Breast Cancer Screening Using Digital Breast Tomosynthesis vs Digital Mammography. JAMA Netw Open. 2020;3(3):e201759.

4. Conant EF, Zuckerman SP, McDonald ES, et al. Five Consecutive Years of Screening with Digital Breast Tomosynthesis: Outcomes by Screening Year and Round. Radiology. 2020 2020:191751.

5. Conant EF, Barlow WE, Herschorn SD, et al. Association of Digital Breast Tomosynthesis vs Digital Mammography With Cancer Detection and Recall Rates by Age and Breast Density. JAMA Oncol. 2019;5(5):635-642.

6. Lourenco AP, Barry-Brooks M, Baird GL, Tuttle A, Mainiero MB. Changes in recall type and patient treatment following implementation of screening digital breast tomosynthesis. Radiology. 2015;274(2):337-342. 
7. Alsheik NH, Dabbous F, Pohlman SK, et al. Comparison of Resource Utilization and Clinical Outcomes Following Screening with Digital Breast Tomosynthesis Versus Digital Mammography: Findings From a Learning Health System. Acad Radiol. 2019;26(5):597-605.

8. Marinovich ML, Hunter KE, Macaskill P, Houssami N. Breast Cancer Screening Using Tomosynthesis or Mammography: A Meta-analysis of Cancer Detection and Recall. J Natl Cancer Inst. 2018;110(9):942-949.

9. Alabousi M, Zha N, Salameh JP, et al. Digital breast tomosynthesis for breast cancer detection: a diagnostic test accuracy systematic review and meta-analysis. Eur Radiol. 2020;30(4):2058-2071.

10. Cochon LR, Giess CS, Khorasani R. Comparing Diagnostic Performance of Digital Breast Tomosynthesis and Full-Field Digital Mammography. J Am Coll Radiol. 2020. 17 (8):999-1003

11. Dang PA, Wang A, Senapati GM, et al. Comparing Tumor Characteristics and Rates of Breast Cancers Detected by Screening Digital Breast Tomosynthesis and Full-Field Digital Mammography. AJR Am J Roentgenol. 2020;214(3):701-706.

12. You C, Zhang Y, Gu Y, et al. Comparison of the diagnostic performance of synthesized two-dimensional mammography and full-field digital mammography alone or in combination with digital breast tomosynthesis. Breast Cancer. 2020;27(1):47-53.

13. Giess CS, Pourjabbar S, Ip IK, Lacson R, Alper E, Khorasani R Comparing Diagnostic Performance of Digital Breast Tomosynthesis and Full-Field Digital Mammography in a Hybrid Screening Environment. AJR Am J Roentgenol. 2017;209(4):929-934.

14. Bahl M, Pinnamaneni N, Mercaldo S, McCarthy AM, Lehman CD. Digital $2 \mathrm{D}$ versus Tomosynthesis Screening Mammography among Women Aged 65 and Older in the United States. Radiology. 2019;291(3):582-590.

15. Rafferty EA, Rose SL, Miller DP, et al. Effect of age on breast cancer screening using tomosynthesis in combination with digital mammography. Breast Cancer Res Treat. 2017;164(3):659-666.

16. Garcia-Albeniz X, Hernan MA, Logan RW, Price M, Armstrong K, Hsu J. Continuation of Annual Screening Mammography and Breast Cancer Mortality in Women Older Than 70 Years. Ann Intern Med. 2020.

17. Siu AL, Force USPST. Screening for Breast Cancer: U.S. Preventive Services Task Force Recommendation Statement. Ann Intern Med. 2016; 164(4):279-296

18. Walter LC, Schonberg MA. Screening mammography in older women: a review. JAMA. 2014;311(13):1336-1347.

19. Fenton JJ, Xing G, Elmore JG, et al. Short-term outcomes of screening mammography using computer-aided detection: a population-based study of medicare enrollees. Ann Intern Med. 2013;158(8):580-587.
20. Fenton JJ, Zhu W, Balch S, Smith-Bindman R, Fishman P, Hubbard RA Distinguishing screening from diagnostic mammograms using Medicare claims data. Med Care. 2014;52(7):e44-51.

21. Koroukian SM, Dahman B, Copeland G, Bradley CJ. The utility of the state buy-in variable in the Medicare denominator file to identify dually eligible Medicare-Medicaid beneficiaries: a validation study. Health Serv Res. 2010;45(1):265-282.

22. Census Tract File for SEER-Medicare Patients. https:// healthcaredelivery.cancer.gov/seermedicare/medicare/tract.census.file. pdf. Published 2015. Accessed 2020.

23. Cancer AJCo. AJCC Cancer Staging Manual. 6th ed. New York: Springer 2002

24. Lannin DR, Wang S. Are Small Breast Cancers Good because They Are Small or Small because They Are Good? $N$ Engl J Med. 2017;376(23):2286-2291.

25. Checka CM, Chun JE, Schnabel FR, Lee J, Toth H. The relationship of mammographic density and age: implications for breast cancer screening. AJR Am J Roentgenol. 2012; 198(3):W292-295.

26. Lowry KP, Coley RY, Miglioretti DL, et al. Screening Performance of Digital Breast Tomosynthesis vs Digital Mammography in Community Practice by Patient Age, Screening Round, and Breast Density. JAMA Netw Open. 2020;3(7):e2011792.

27. Lee CI, Cevik M, Alagoz O, et al. Comparative effectiveness of combined digital mammography and tomosynthesis screening for women with dense breasts. Radiology. 2015;274(3):772-780.

28. Lowry KP, Trentham-Dietz A, Schechter CB, et al. Long-Term Outcomes and Cost-Effectiveness of Breast Cancer Screening With Digital Breast Tomosynthesis in the United States. J Natl Cancer Inst. 2020;112(6):582589.

29. Schonberg MA, Marcantonio ER, Ngo L, Li D, Silliman RA, McCarthy EP. Causes of death and relative survival of older women after a breast cancer diagnosis. J Clin Oncol. 2011;29(12):1570-1577.

30. Cronin PA, Olcese C, Patil S, Morrow M, Van Zee KJ. Impact of Age on Risk of Recurrence of Ductal Carcinoma In Situ: Outcomes of 2996 Women Treated with Breast-Conserving Surgery Over 30 Years. Ann Surg Oncol. 2016;23(9):2816-2824.

Publisher's Note Springer Nature remains neutral with regard to jurisdictional claims in published maps and institutional affiliations. 